\title{
Porting OpenMP to GPGPU to accelerate Krylov space computations in coupled geodynamics simulator
}

\author{
Saumik Dana \\ University of Southern California \\ Los Angeles, CA 90007 \\ sdana@usc.edu
}

\begin{abstract}
Understanding the causality between the events leading upto and post fault slip and the earthquake recording is important for seismic design and monitoring of underground structures, bridges and reinforced concrete buildings as well as climate mitigation projects like carbon sequestration and energy technologies like enhanced geothermal systems or oilfield wastewater disposal. While the events leading upto fault slip are typically governed by poroelastostatics, the events post fault slip can easily transition into poroelastodynamics territory due to runaway fault slip velocities. An understanding of expected fault slip velocities is critical apriori, as an algorithm which can seamlessly transition from time marching in poroelastostatics realm to poroelastodynamics realm and vice-versa is extremely difficult to achieve. That being said, every effort in the direction of accelerating the computations on the flow side are a necessary step forward in rendering a fast coupled geodynamics simulator. In this document, we present a framework in which we study the porting of the OpenMP parallelism of the flow simulator to a GPGPU
\end{abstract}

\section{Introduction}

The earthquake cycle, from slow deformation associated with interseismic behavior to rapid deformation associated with earthquake rupture, spans spatial scales ranging from fractions of a meter associated with the size of contact asperities on faults and individual grains to hundreds of kilometers associated with plate boundaries [1]. Similarly, temporal scales range from fractions of a second associated with slip at a point during earthquake rupture to hundreds of years of strain accumulation between earthquakes. In many cases, earthquakes are triggered after pore pressure perturbations activate critically stressed seismogenic faults [1], not just due to natural causes like earth tides [2], rainfall [3], snowfall [4], typhoons [5], but also due to human activity [6]. As faults slip, the induced stress field spawns seismic waves, which travel through and around the earth and can be recorded with seismometers. There are several different kinds of seismic waves, and they all move in different ways. The two main types of waves are body waves (P- and S-) and surface waves. Body waves can travel through the Earth's inner layers, but surface waves can only move along the surface of the planet like ripples on water. Understanding the causality between the events leading upto and post fault slip and the earthquake recording is important for seismic design and monitoring of underground structures [7], bridges [8] and reinforced concrete buildings [9] as well as climate mitigation projects like carbon sequestration [10] and energy technologies like enhanced geothermal systems [11] or oilfield wastewater disposal [12]. 
The complexity of the many physical processes operating over this vast range of scales leads most researchers to focus on a narrow space-time window to isolate just one or a few processes; the limited spatial and temporal coverage of observations also often justifies this narrow focus. For quasi-static simulations, the inertial term is ignored and time dependence only enters through the constitutive models and the loading conditions. As a result, a quasi-static simulation is the solution to a series of static problems with potentially time-varying physical properties and boundary conditions. In dynamic simulations, we shall include the inertial term to resolve the propagation of seismic waves, with an intended focus on applications for earthquake physics and ground-motion simulations. The only place where things can accelerate fast is on the fault and the only time it happens is when fault slips seismically $(>1 \mathrm{~cm} / \mathrm{sec})$. If the fault slip is aseismic or creeping $(<0.1 \mathrm{~mm} / \mathrm{sec})$, then accelerations are negligible and quasi-static and quasi-dynamic [13] approximations are valid. In our models, we know the fault is the location where things will happen, but we do not know a priori is whether it will be seismic, aseismic or in the transition region. While back-of-the-envelope estimations can be done from the strain energy accumulated in the rock surrounding the faults, the fault's friction properties, and the rock's elastic moduli, the more optimal and accurate option is to use the simulator to do this estimation by equipping it with the physics of elastodynamics.

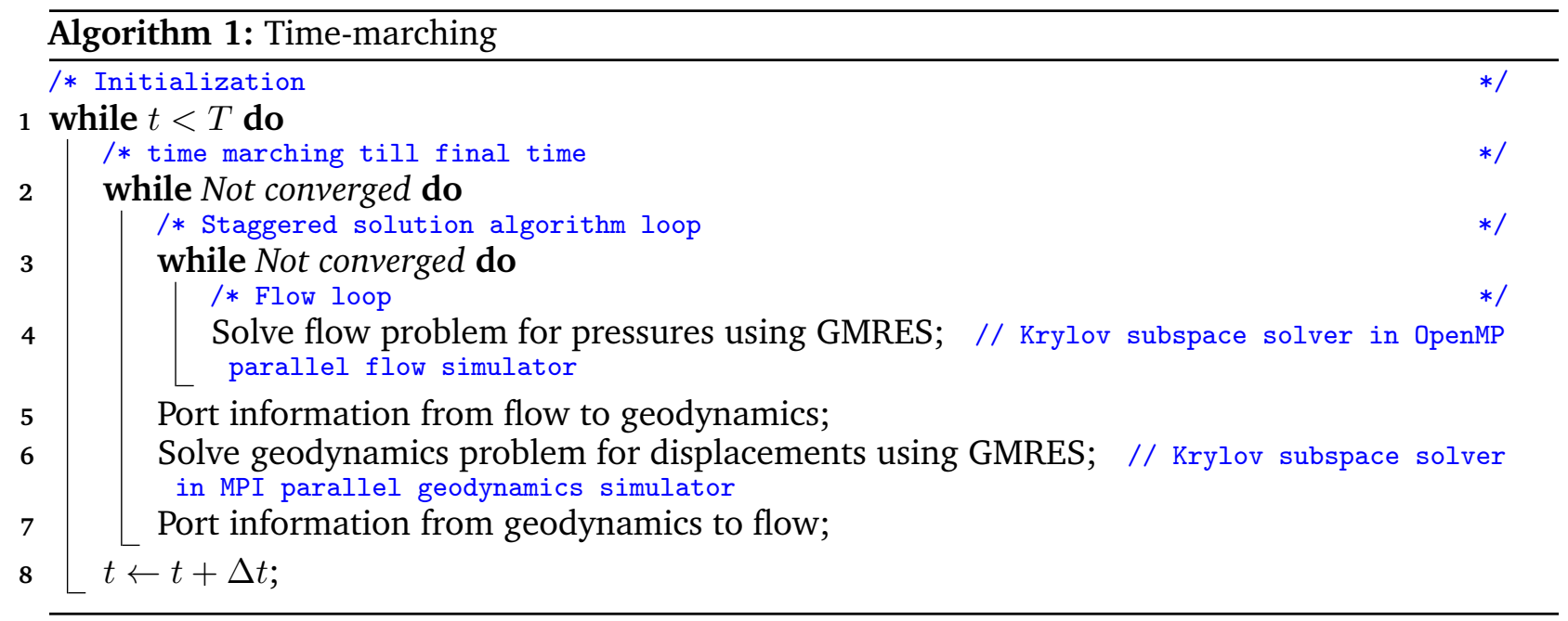

The simulator is a mixed programming language hybrid MPI and OpenMP framework in which the open source geodynamics code $[14,15]$ written in Python and C ++ is MPI-based and the in-house multiphase/multicomponent flow code written in $\mathrm{C}++$ is OpenMP-based [16]. As shown in Algorithm 1, the framework follows the popular staggered solution algorithm which allows for different simulators written in separate languages with respective parallelism frameworks are coupled together to render a coupled simulator [16-26]. There are three layers in which the code framework operates out of separate directories

$\checkmark$ The high-level Python code in the geodynamics module makes use of Pyre [27] to link the sub-modules together at runtime

$\checkmark$ The low-level $\mathrm{C}++$ code performs operations on matrices and vectors in parallel using the Standard Template Library [28]

$\checkmark$ The SWIG [29] code with all the .i files, and SWIG-generated python code and MODULENAME_wrap.cxx which wraps the Python code around the $\mathrm{C}++$ code to create python modules.

Currently, the code base is hybrid MPI and OpenMP, in which the geodynamics code is MPI-parallel and the flow code is OpenMP-parallel. This hybrid framework, although accurate, brings in efficiency issues for large size simulations. While the geodynamics elements are distributed among MPI processes with each process running the same tasks on a different sub-grids of the main grid, the 
flow elements are all stacked together in the root process, and the tasks are distributed among threads in the root process. When the data structures port information from the geodynamics code to the flow code, they are assembled into the root process using the MPI Gather command, and then transfered to the flow simulator. This porting can happen multiple times in each time step depending on the convergence requirements of the staggered solution algorithm, and this adds inefficiency to the overall computational run-time. The first option to increase efficiency is to port the OpenMP parallel functionality to MPI parallel [30] on the flow side, and the second option is to offload the OpenMP parallel computations onto a GPGPU [31].

The second option is definitely more engaging as it leverages the massive computational power of the GPGPU. That being said, the advent of new, massively parallel architectures [32] has greatly increased the penalty for suboptimal non-vectorized code while providing much more energy efficient performance. They also increase the imbalance between flop rate and memory bandwidth or latency, so that thousands of flops can be needed to cover outstanding memory references. GPUs in particular have very high memory latency coupled with a wide bus, making the memory access pattern critical for good performance. In addition, the size of fast cache memory per core has shrunk dramatically, so that it cannot easily be used to hide irregular memory access. We explore the second option in this document.

\section{Governing equations}

\subsection{Solid subsystem}

The governing equation for linear momentum balance of the solid system can be expressed as

$$
\nabla \cdot \boldsymbol{\sigma}+\rho_{b} \boldsymbol{g}=\rho_{b} \ddot{\boldsymbol{u}}
$$

where $\boldsymbol{\sigma}$ is the Cauchy total stress tensor, $\boldsymbol{g}$ is the gravity vector, and $\rho_{b}=\phi \sum_{\beta}^{n_{\text {phase }}} \rho_{\beta} S_{\beta}+(1-\phi) \rho_{s}$ is the bulk density, $\rho_{\beta}$ and $S_{\beta}$ are the density and saturation of fluid phase $\beta, \rho_{s}$ is the density of the solid phase, $\phi$ is the true (Eulerian) porosity, and $n_{\text {phase }}$ is the number of fluid phases. In the quasi-static case, the inertial term $\rho_{b} \ddot{\boldsymbol{u}}$ is ignored. Note that, by definition, the sum of all fluid phase saturations satisfies $\sum_{\beta}^{n_{\text {phase }}} S_{\beta} \equiv 1$. Following [33], the poroelasticity equations can be written in incremental form as

$$
\delta \boldsymbol{\sigma}=\boldsymbol{C}_{d r}: \delta \varepsilon-b \delta \tilde{p} \mathbf{1} \equiv \boldsymbol{C}_{d r}: \delta \varepsilon-\sum_{\beta} b_{\beta} p_{\beta} \mathbf{1},
$$

where $\tilde{p}=\sum_{\beta} S_{\beta} p_{\beta}$, and $b_{\beta}=b S_{\beta}$ are the Biot coefficients for individual phases such that $\sum_{\beta} b_{\beta}=b$, $\boldsymbol{C}_{d r}$ is the rank-4 drained elasticity tensor, $\mathbf{1}$ is the rank-2 identity tensor, $b$ is the Biot coefficient of the saturated porous material, $\varepsilon$ is the linearized strain tensor, defined as the symmetric gradient of the displacement vector $\boldsymbol{u}$,

$$
\varepsilon:=\frac{1}{2}\left(\nabla \boldsymbol{u}+\nabla^{\mathrm{T}} \boldsymbol{u}\right) \equiv \frac{1}{3} \varepsilon_{v} \mathbf{1}+\boldsymbol{e},
$$

where $\varepsilon_{v}=\operatorname{tr}(\varepsilon)$ is the volumetric strain and $e$ is the deviatoric strain tensor. The increment in mean total stress is given by

$$
d \sigma_{v}=\frac{1}{3} \operatorname{tr}(\delta \boldsymbol{\sigma})=\frac{1}{3} \operatorname{tr}\left(\boldsymbol{C}_{d r}: \delta \boldsymbol{\varepsilon}-b \delta \tilde{p} \mathbf{1}\right)=K_{d r} d \varepsilon_{v}-b \delta \tilde{p}
$$

where $K_{d r}$ is drained bulk modulus of porous medium. Eq. (4) can also be written as

$$
d \varepsilon_{v}=\frac{d \sigma_{v}}{K_{d r}}+\frac{b d \tilde{p}}{K_{d r}}
$$

From the solid mass conservation equation, the incremental porosity update is given as $[34,35]$

$$
d \phi=\left(\frac{b-\phi}{K_{d r}}\right)\left(d \sigma_{v}+d \tilde{p}\right)
$$




\subsection{Fluid subsystem}

Assuming that the fluids are immiscible, the mass-conservation equation for each phase $\alpha$ is

$$
\frac{d m_{\alpha}}{d t}+\nabla \cdot \boldsymbol{w}_{\alpha}=\rho_{\alpha} f_{\alpha}
$$

where the accumulation term $d m_{\alpha} / d t$ describes the time variation of fluid mass relative to the motion of the solid skeleton, $\boldsymbol{w}_{\alpha}$ is the mass-flux of fluid phase $\alpha$ relative to the solid skeleton, and $f_{\alpha}$ is the volumetric source term for phase $\alpha$ that can model the effect of wells. In the deformed configuration, the mass of phase $\alpha$ per unit volume of porous medium is

$$
m_{\alpha}=\rho_{\alpha} S_{\alpha} \phi J
$$

where $J=1+\varepsilon_{v}$ is the Jacobian of deformation under infinitesimal deformation theory. The incremental fluid mass can be written as

$$
\frac{d m_{\alpha}}{\rho_{\alpha}}=\phi J \frac{\partial S_{\alpha}}{\partial P_{\alpha \beta}} d P_{\alpha \beta}+\phi J S_{\alpha} c_{\alpha} d p_{\alpha}+\phi S_{\alpha} d \varepsilon_{v}+J S_{\alpha} d \phi,
$$

where $c_{\alpha}=\frac{1}{\rho_{\alpha}} \frac{d \rho_{\alpha}}{d p_{\alpha}}$ is the compressibility of the fluid phase $\alpha, P_{\alpha \beta}=p_{\beta}-p_{\alpha}$ is the capillary pressure and $\partial S_{\alpha} / \partial P_{\alpha \beta}$ is the inverse capillary pressure derivative which can be obtained from one of the $P_{\alpha \beta}$ vs. $S_{\alpha}$ models commonly used in flow simulations, e.g. the van Genuchten model. Substituting Eqs. (6) and (5) in Eq. (8) and assuming $\varepsilon_{v} \ll 1$ and noting that $b_{\alpha}=b S_{\alpha}$, we get

$$
\frac{d m_{\alpha}}{\rho_{\alpha}}=\phi \frac{\partial S_{\alpha}}{\partial P_{\alpha \beta}} d P_{\alpha \beta}+\phi S_{\alpha} c_{\alpha} d p_{\alpha}+N S_{\alpha} \sum_{\beta} S_{\beta} \delta p_{\beta}+b^{2} \frac{S_{\alpha}}{K_{d r}} \sum_{\beta} S_{\beta} \delta p_{\beta}+\frac{b_{\alpha} d \sigma_{v}}{K_{d r}}
$$

where $N=(b-\phi)(1-b) / K_{d r}$. Defining $N_{\alpha \beta}$ from the first three terms on the right hand side and substituting the above in Eq. (7), we obtain the multiphase flow equation for phase $\alpha$ in a poroelastic medium as:

$$
\frac{\partial}{\partial t}\left(\rho_{\alpha} \sum_{\beta}\left(N_{\alpha \beta}+\frac{b_{\alpha} b_{\beta}}{K_{d r}}\right) p_{\beta}\right)+\frac{1}{K_{d r}} \frac{\partial}{\partial t}\left(\rho_{\alpha} b_{\alpha} \sigma_{v}\right)+\nabla \cdot \boldsymbol{w}_{\alpha}=\rho_{\alpha} f_{\alpha}
$$

Because we assume that the fluids are immiscible, the mass-flux of phase $\alpha$ is $\boldsymbol{w}_{\alpha}=\rho_{\alpha} \boldsymbol{v}_{\alpha}$, where we adopt the traditional multiphase-flow extension of Darcy's law [36, 37]:

$$
\boldsymbol{v}_{\alpha}=-\frac{\boldsymbol{k} k_{\alpha}^{r}}{\mu_{\alpha}}\left(\nabla p_{\alpha}-\rho_{\alpha} \boldsymbol{g}\right)
$$

where $\mu_{\alpha}$ and $k_{\alpha}^{r}$ are the dynamic viscosity and the relative permeability of phase $\alpha$ in presence of other fluid phases.

\section{OpenMP to GPGPU}

Data parallelism is a form of parallelization of computing across multiple processors in parallel computing environments. It mainly focuses on distributing the data across different parallel computing nodes. In a multiprocessor system executing a single set of instructions (SIMD), data parallelism is achieved when each processor performs the same task on different pieces of distributed data. In some situations, a single execution thread controls operations on all pieces of data. In others, different threads control the operation, but they execute the same code. 


\subsection{OpenMP programming and memory model}

OpenMP is an implementation of multithreading, a method of parallelizing whereby a master thread (a series of instructions executed consecutively) forks a specified number of slave threads and the system divides a task among them. The threads then run concurrently, with the runtime environment allocating threads to different processors. The section of code that is meant to run in parallel is marked accordingly, with a preprocessor directive that will cause the threads to form before the section is executed. Each thread has an id attached to it which can be obtained using a function (called omp_get_thread_num()). The thread id is an integer, and the master thread has an id of 0 . After the execution of the parallelized code, the threads join back into the master thread, which continues onward to the end of the program. By default, each thread executes the parallelized section of code independently. Work-sharing constructs can be used to divide a task among the threads so that each thread executes its allocated part of the code. Both task parallelism and data parallelism can be achieved using OpenMP in this way. The runtime environment allocates threads to processors depending on usage, machine load and other factors. The runtime environment can assign the number of threads based on environment variables, or the code can do so using functions. The OpenMP functions are included in a header file labeled omp.h in $\mathrm{C} / \mathrm{C}++$. One might expect to get an $\mathrm{N}$ times speedup when running a program parallelized using OpenMP on a $\mathrm{N}$ processor platform. However, this seldom occurs for these reasons:

$\checkmark$ When a dependency exists, a process must wait until the data it depends on is computed.

$\checkmark$ When multiple processes share a non-parallel proof resource (like a file to write in), their requests are executed sequentially. Therefore each thread must wait until the other thread releases the resource.

$\checkmark$ A large part of the program may not be parallelized by OpenMP, which means that the theoretical upper limit of speedup is limited according to Amdahl's law.

$\checkmark \mathrm{N}$ processors in a symmetric multiprocessing (SMP) may have $\mathrm{N}$ times the computation power, but the memory bandwidth usually does not scale up N times. Quite often, the original memory path is shared by multiple processors and performance degradation may be observed when they compete for the shared memory bandwidth.

Many other common problems affecting the final speedup in parallel computing also apply to OpenMP, like load balancing and synchronization overhead.

\subsection{CUDA programming and memory model}

The CUDA programming model [38] is a general-purpose multi-threaded SIMD model for GPGPU programming. A GPU is viewed as a parallel computing coprocessor, which can execute a large number of threads concurrently. A CUDA program consists of a series of sequential and parallel execution phases. Sequential phases have little or no parallelism, and thus they are executed on the CPU as host code. Parallel phases that exhibit rich data parallelism are implemented as a set of kernel functions, which are executed on the GPU. Each kernel function specifies GPU code to be executed in an SIMD fashion, by a number of threads invoked for each parallel phase. In the CUDA programming model, a host CPU and a GPU device have separate address spaces. For a CPU to access GPU data, the CUDA model provides an API for explicit GPU memory management, including functions to transfer data between a CPU and a GPU.

The CUDA memory model has

$\checkmark$ an off-chip global memory space, which is accessible by all threads,

$\checkmark$ an off-chip local memory space, which is private to each thread,

$\checkmark$ a fast on-chip shared memory space, which is shared only by threads in the same thread block,

$\checkmark$ and registers, which are private to each thread

$\checkmark$ constant memory with a dedicated small cache for read-only global data 
$\checkmark$ texture memory with a dedicated small cache for read-only array data accessed through built-in texture functions

The shared memory and the register bank in a multiprocessor are dynamically partitioned among the active thread blocks running on the multiprocessor. Therefore, register and shared memory usages per thread block can be a limiting factor preventing full utilization of execution resources.

\subsection{OpenMP to CUDA}

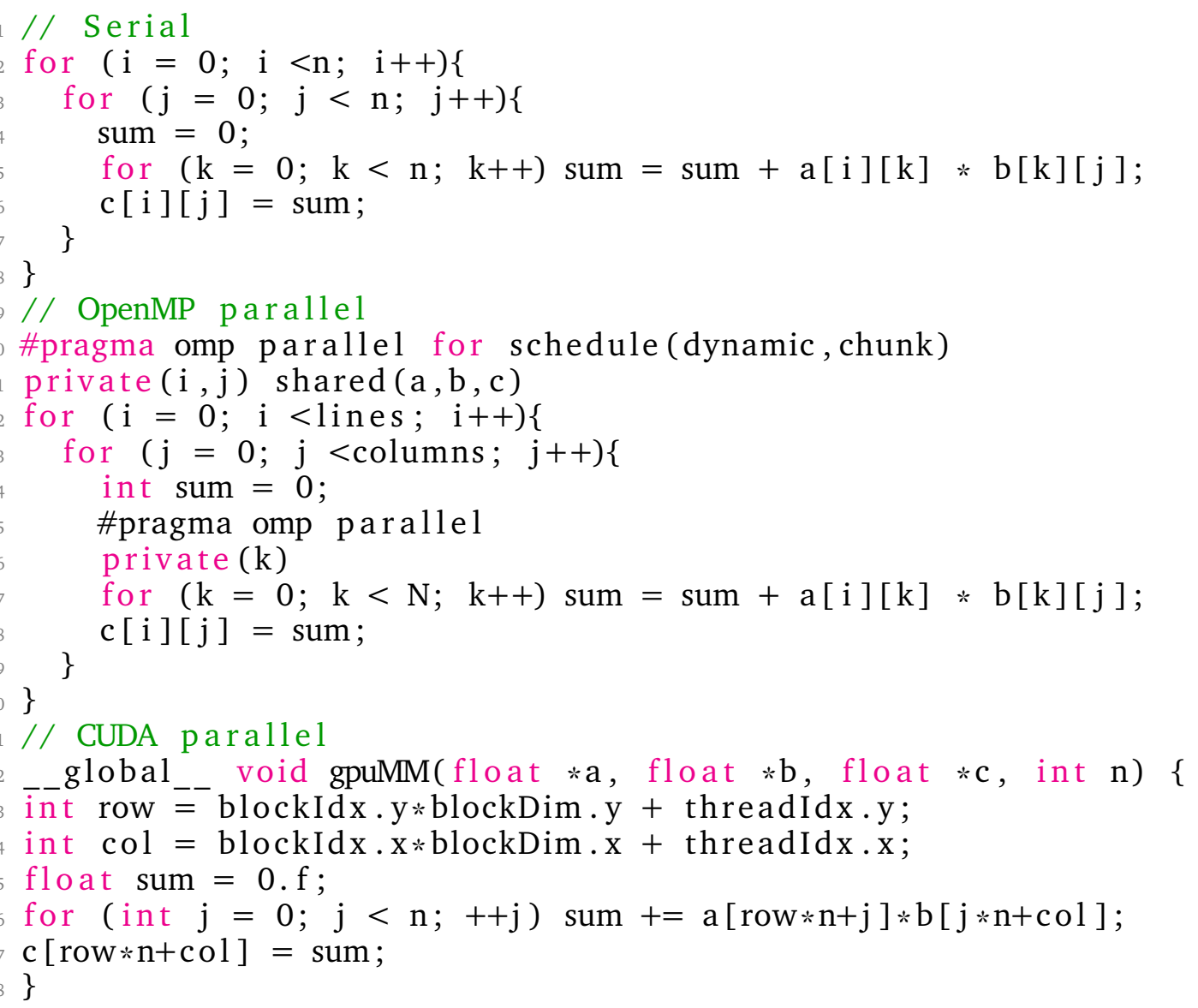

Listing 1: Different versions of matrix matrix multiplication

Code snippets for serial, OpenMP parallel and CUDA parallel for archetypal matrix matrix multiplication are given in Listings 1 . There are several advantages of OpenMP as a programming paradigm for GPGPUs

$\checkmark$ OpenMP is efficient at expressing loop-level parallelism in applications, which is an ideal target for utilizing GPU's highly parallel computing units to accelerate data-parallel computations

$\checkmark$ The concept of a master thread and a pool of worker threads in OpenMP 's fork-join model represents well the relationship between the master thread running in a host CPU and a pool of threads in a GPU device

$\checkmark$ Incremental parallelization of applications, which is one of OpenMP's features, can add the same benefit to GPGPU programming 


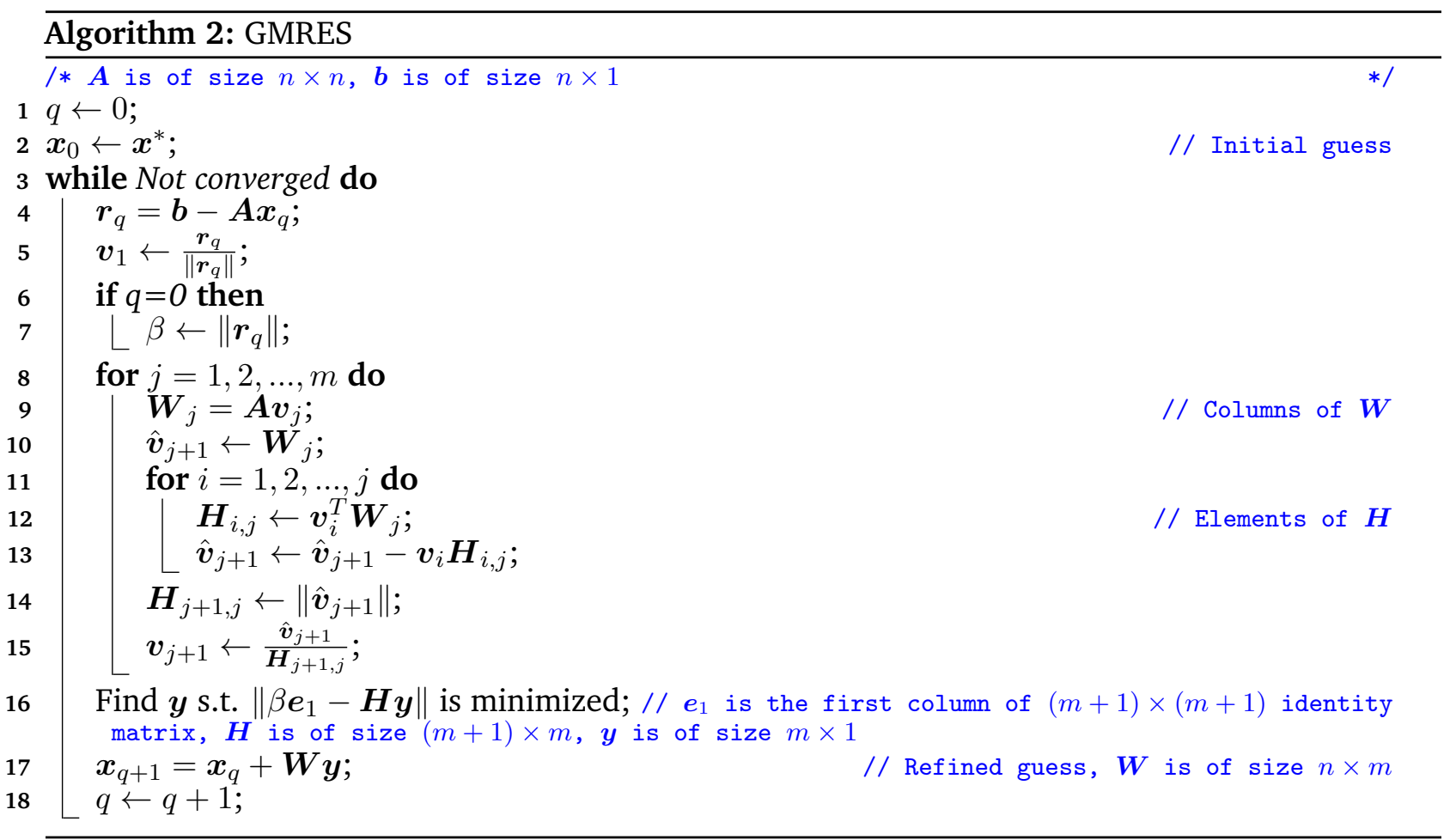

\subsection{Krylov subspace solver and OpenMP implementation}

The governing PDEs (Eqs. (1) and (9)) are discretized using finite element method and finite volume method respectively, and the resulting system of algebraic equations is solved using Krylov space methods [39-42], which generate a sequence of iterates $x_{n}$ that are approximate solutions. In these algorithms, $x_{n}$ belongs to an affine space that grows with $n$ :

$$
\boldsymbol{x}_{n}-\boldsymbol{x}_{0} \in \operatorname{span}\left(\boldsymbol{r}_{0}, \boldsymbol{A} \boldsymbol{r}_{0}, \cdots, \boldsymbol{A}^{n-1} \boldsymbol{r}_{0}\right)
$$

where $\boldsymbol{r}_{0}:=\boldsymbol{b}-\boldsymbol{A} \boldsymbol{x}_{0}$ is the initial residual. The algebraic system of equations resulting from the flow equations (Eqs. (9)) are solved using GMRES [42] as shown in Algorithm 2. In a nutshell, the residuals at each iteration are QR-factorized, and guesses are refined using the solution of a least-squares problem.

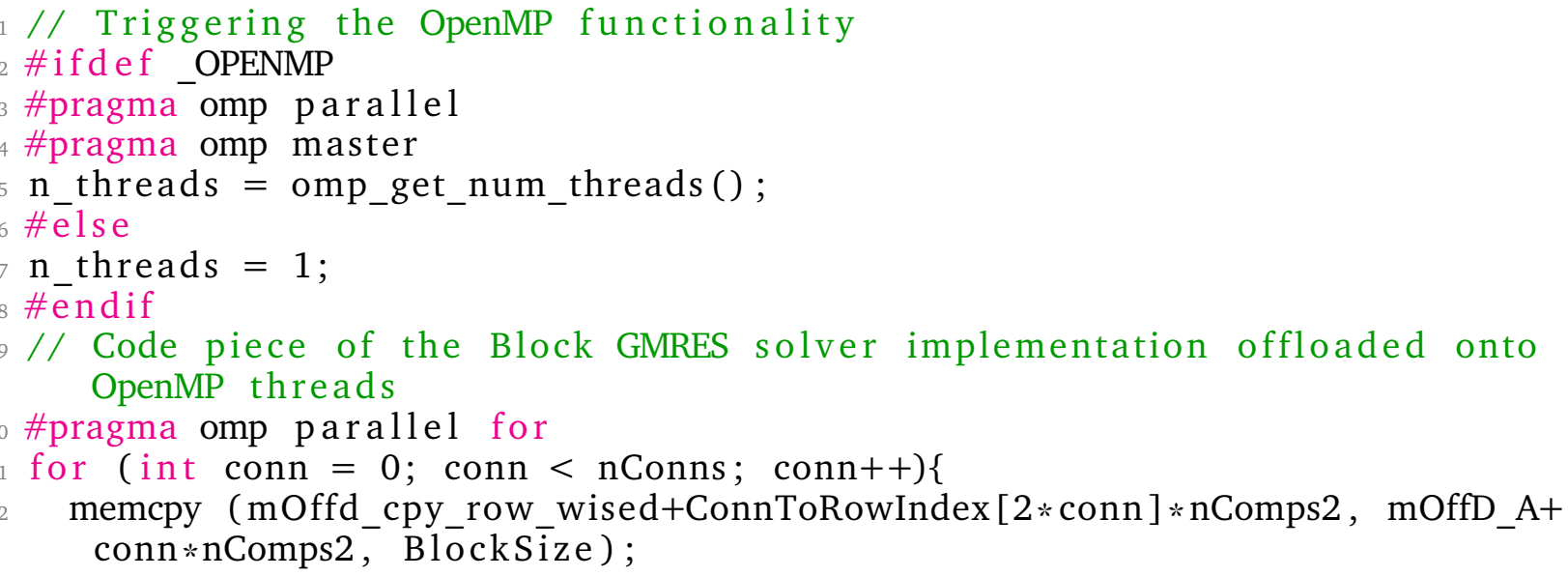


13 \}

\section{Listing 2: Triggering the OpenMP functionality}

The code snippets in the flow simulator that have evidence of OpenMP usage are provided in Listings 2.

\section{Conclusions and Outlook}

Due to the similarity between OpenMP and CUDA programming models, we should be able to convert OpenMP parallelism, especially loop-level parallelism, into the forms that best express parallelism in CUDA. However, the baseline translation of existing OpenMP programs does not always yield good performance. Performance gaps are due to architectural differences between traditional shared-memory multi-processors (SMPs), served by OpenMP, and stream architectures, adopted by most GPUs. Even though the OpenMP programming model is platform-independent, most existing OpenMP programs were tuned to traditional shared-memory multiprocessors. We refer to stream architectures as those that operate on a large data space (or stream) in parallel, typically in an SIMD manner and tuned for fast access to regular, consecutive elements of the data stream. In GPU architectures, optimization techniques designed for CPU-based algorithms may not perform well [7]. Also, GPUs face bigger challenges in handling irregular applications than SMPs, because of the stream architectures' preference for regular access patterns. Handling all these issues is part of future work.

\section{References}

[1] Hiroo Kanamori and Emily E Brodsky. The physics of earthquakes. Reports on Progress in Physics, 67(8):1429, 2004.

[2] Christopher H Scholz, Yen Joe Tan, and Fabien Albino. The mechanism of tidal triggering of earthquakes at mid-ocean ridges. Nature communications, 10(1):1-7, 2019.

[3] Sebastian Hainzl, Toni Kraft, Joachim Wassermann, Heiner Igel, and E Schmedes. Evidence for rainfall-triggered earthquake activity. Geophysical Research Letters, 33(19), 2006.

[4] EK Montgomery-Brown, David R Shelly, and Paul A Hsieh. Snowmelt-triggered earthquake swarms at the margin of long valley caldera, california. Geophysical Research Letters, 46(7):3698-3705, 2019.

[5] ChiChing Liu, Alan T Linde, and I Selwyn Sacks. Slow earthquakes triggered by typhoons. Nature, 459(7248):833-836, 2009.

[6] Gillian R Foulger, Miles P Wilson, Jon G Gluyas, Bruce R Julian, and Richard J Davies. Global review of human-induced earthquakes. Earth-Science Reviews, 178:438-514, 2018. 
Porting OpenMP to GPGPU to accelerate Krylov space computations in coupled geodynamics simulator

[7] Youssef MA Hashash, Jeffrey J Hook, Birger Schmidt, I John, and Chiang Yao. Seismic design and analysis of underground structures. Tunnelling and underground space technology, 16(4):247-293, 2001.

[8] MJ Nigel Priestley, Frieder Seible, and Gian Michele Calvi. Seismic design and retrofit of bridges. John Wiley \& Sons, 1996.

[9] Jack Moehle. Seismic design of reinforced concrete buildings. McGraw-Hill Education, 2015.

[10] Mark D Zoback and Steven M Gorelick. Earthquake triggering and large-scale geologic storage of carbon dioxide. Proceedings of the National Academy of Sciences, 109(26):10164-10168, 2012.

[11] William L Ellsworth, Domenico Giardini, John Townend, Shemin Ge, and Toshihiko Shimamoto. Triggering of the pohang, korea, earthquake ( $\mathrm{m} \mathrm{w} \mathrm{5.5)} \mathrm{by} \mathrm{enhanced} \mathrm{geothermal} \mathrm{system}$ stimulation. Seismological Research Letters, 90(5):1844-1858, 2019.

[12] Katie M Keranen and Matthew Weingarten. Induced seismicity. Annual Review of Earth and Planetary Sciences, 46:149-174, 2018.

[13] J. R. Rice. Spatio-temporal complexity of slip on a fault. J. Geophys. Res., 98:9885-9907, 1993.

[14] B Aagaard, S Kientz, M Knepley, L Strand, and C Williams. Pylith user manual: version 2.1. 0. Davis, CA: Computational Infrastructure of Geodynamics, 2013.

[15] Brad T Aagaard, Matthew G Knepley, and Charles A Williams. A domain decomposition approach to implementing fault slip in finite-element models of quasi-static and dynamic crustal deformation. Journal of Geophysical Research: Solid Earth, 118(6):3059-3079, 2013.

[16] Birendra Jha and Ruben Juanes. Coupled multiphase flow and poromechanics: A computational model of pore pressure effects on fault slip and earthquake triggering. Water Resources Research, 50(5):3776-3808, 2014.

[17] Saumik Dana, Xiaoxi Zhao, and Birendra Jha. A two-grid computational framework for fast monitoring of fault stability and ground deformation in multiphase geomechanics. Under review at Journal of Computational Physics, 2021.

[18] Saumik Dana and Mary F Wheeler. Design of convergence criterion for fixed stress split iterative scheme for small strain anisotropic poroelastoplasticity coupled with single phase flow. arXiv preprint arXiv:1912.06476, 2019.

[19] Saumik Dana. System of equations and staggered solution algorithm for immiscible two-phase flow coupled with linear poromechanics. arXiv preprint arXiv:1912.04703, 2019.

[20] Saumik Dana, Joel Ita, and Mary F Wheeler. The correspondence between voigt and reuss bounds and the decoupling constraint in a two-grid staggered algorithm for consolidation in heterogeneous porous media. Multiscale Modeling \& Simulation, 18(1):221-239, 2020.

[21] Saumik Dana, Xiaoxi Zhao, and Birendra Jha. Two-grid method on unstructured tetrahedra: Applying computational geometry to staggered solution of coupled flow and mechanics problems. arXiv preprint arXiv:2102.04455, 2021.

[22] Saumik Dana and Birendra Jha. A fault slip model to study earthquakes due to pore pressure perturbations. arXiv preprint arXiv:2104.06257, 2021.

[23] S. Dana and M. F. Wheeler. Convergence analysis of fixed stress split iterative scheme for anisotropic poroelasticity with tensor biot parameter. Computational Geosciences, 22(5):12191230, 2018.

[24] S. Dana and M. F. Wheeler. Convergence analysis of two-grid fixed stress split iterative scheme for coupled flow and deformation in heterogeneous poroelastic media. Computer Methods in Applied Mechanics and Engineering, 341:788-806, 2018.

[25] Saumik Dana, Benjamin Ganis, and Mary F. Wheeler. A multiscale fixed stress split iterative scheme for coupled flow and poromechanics in deep subsurface reservoirs. Journal of Computational Physics, 352:1-22, 2018. 
[26] S. Dana. Addressing challenges in modeling of coupled flow and poromechanics in deep subsurface reservoirs. PhD thesis, The University of Texas at Austin, 2018.

[27] K Jarrod Millman and Michael Aivazis. Python for scientists and engineers. Computing in Science \& Engineering, 13(2):9-12, 2011.

[28] Nicolai M Josuttis. The C++ standard library: a tutorial and reference. Addison-Wesley, 2012.

[29] Teresa L Cottom. Using swig to bind c++ to python. Computing in Science \& Engineering, 5(2):88-97, 2003.

[30] Ayon Basumallik and Rudolf Eigenmann. Towards automatic translation of openmp to mpi. In Proceedings of the 19th annual international conference on Supercomputing, pages 189-198, 2005.

[31] Seyong Lee, Seung-Jai Min, and Rudolf Eigenmann. Openmp to gpgpu: a compiler framework for automatic translation and optimization. ACM Sigplan Notices, 44(4):101-110, 2009.

[32] Craig M Wittenbrink, Emmett Kilgariff, and Arjun Prabhu. Fermi gf100 gpu architecture. IEEE Micro, 31(2):50-59, 2011.

[33] O. Coussy. Mechanics of Porous Continua. John Wiley and Sons, Chichester, England, 1995.

[34] J. Geertsma. The effect of fluid pressure decline on volumetric changes of porous rocks. SPE, 210:331-340, 1957.

[35] R. J. S. Brown and J. Korringa. On the dependence of the elastic properties of a porous rock on the compressibility of the pore fluid. Geophysics, 40(4):608-616, 1975.

[36] M. Muskat. Physical Principles of Oil Production. McGraw-Hill, New York, 1949.

[37] J. Bear. Dynamics of Fluids in Porous Media. Wiley, New York, 1972.

[38] Jason Sanders and Edward Kandrot. CUDA by example: an introduction to general-purpose GPU programming. Addison-Wesley Professional, 2010.

[39] Yousef Saad. Iterative Methods for Sparse Linear Systems. SIAM, 2003.

[40] Allison H Baker, John M Dennis, and Elizabeth R Jessup. On improving linear solver performance: A block variant of gmres. SIAM Journal on Scientific Computing, 27(5):1608-1626, 2006.

[41] Rudnei Dias da Cunha and Dulcenéia Becker. Dynamic block gmres: an iterative method for block linear systems. Advances in Computational Mathematics, 27(4):423-448, 2007.

[42] Youcef Saad and Martin H Schultz. Gmres: A generalized minimal residual algorithm for solving nonsymmetric linear systems. SIAM Journal on scientific and statistical computing, 7(3):856-869, 1986. 